\title{
Novel patented markers of immune checkpoint inhibitor efficacy
}

\author{
Matthieu Collin*,1 \\ ${ }^{1}$ Inserm Transfert, 7 Rue Watt, 75013 Paris, France \\ *Author for correspondence: matthieu.collin@inserm-transfert.fr
"Immunotherapy in cancer is facing a revolution with the advent of immune-checkpoint inhibitors (ICI)."

First draft submitted: 26 February 2018; Accepted for publication: 12 March 2018; Published online: 8 June 2018

Keywords: biomarkers $\bullet$ immune checkpoint inhibitors $\bullet$ tumor microenvironment $\bullet$ gene $\bullet$ immunoprofiling

Immunotherapy in cancer is facing a revolution with the advent of immune-checkpoint inhibitors (ICI). Activation of $\mathrm{T}$ cells is indeed regulated by the immune checkpoints acting as co-stimulatory or co-inhibitory molecules expressed on $\mathrm{T}$ cells that interact with their co-ligands expressed in different cells present in the tumor microenvironment (TME) [1]. These include the cancer cells themselves but also other immune cells (e.g., monocytic cells), endothelial and stroma cells. One of the most extensively studied co-inhibitory molecule is PD-1 and its ligand PD-L1. Many antibody constructs have been developed to block this interaction and were approved during the last few years. For instance, the US FDA has already approved anti-PD-1 monoclonal antibodies including nivolumab $\left(\right.$ Opdivo $\left.^{\circledR}\right)$ and pembrolizumab (Keytruda ${ }^{\circledR}$ ) and anti-PD-L1 antibodies such as atezolizumab (Tecentriq ${ }^{\circledR}$ ), avelumab (Bavencio $\left.{ }^{\circledR}\right)$, and durvalumab (Imfinzi $\left.{ }^{\circledR}\right)$. The drugs have indeed shown significant therapeutic activity in various cancers [2]. Further to these successes, the number of approvals for ICIs are rapidly increasing. However, the efficacy of the immune checkpoint blockade therapy (ICBT) varies greatly in patients and among different tumor types. Therefore, the development of biomarkers for response to ICI is of particular interest, in order to identify, preferably before initiation of treatment, which patients are likely to achieve a response to such treatments. The most well-studied biomarker is PD-L1 protein expression in the TME and is currently the only FDA-approved companion biomarker of ICIs. However, there are well-recognized limitations for this predictive biomarker and thus a substantial unmet need remains.

\section{Marker based on immunoprofiling}

The quantity, quality, and distribution of immune cells in the TME justifies the concept of using immunoprofiling to predict the response to therapy, in particular to ICBT. For instance, recent reports indicate that the presence of $\mathrm{CD}^{+} \mathrm{T}$ cells expressing PD-L1 could predict a response to anti-PD-L1 treatment, and a global increase of the $\mathrm{CD}^{+}$T-cell density was observed after treatment in responding tumors [3]. In this context, measuring the expression of at least one gene representative of human adaptive immune response (e.g., CD8) and at least one gene representative of the immunosuppressive response (e.g., PD-L1) could be suitable for predicting the response to ICIs [4]. Driven by this concept, different immune signatures have also been developed for predicting the ICI efficacy. In particular, a patent claiming a combination of at least seven genes selected from the group consisting of CCR2, CD3D, CD3E, CD3G, CD8A, CXCL10, CXCL11, GZMA, GZMB, GZMK, GZMM, IL15, IRF1, PRF1, STAT1, CD69, ICOS, CXCR3, STAT4, CCL2 and TBX21 for predicting survival time and response to ICIs was recently granted by the European Patent Office [5].

Within the TME, immune cells may also have a dark side linked to the maintenance of a deleterious chronic inflammation, which has been shown to promote carcinogenesis and to hamper the efficiency of tumor immune surveillance. Recent results obtained in lung cancers indeed suggest that a co-existing chronic obstructive pulmonary disease is associated with an increased sensibility of tumor infiltrating lymphocytes to mechanisms developed by malignant cells to avoid tumor immune surveillance and may indicate a higher sensitivity to immune checkpoint 
blockade therapies. On this basis, an international patent application was recently filed with claims relating to methods of predicting whether a patient suffering from a non-small cell lung cancer will achieve a response with an immune checkpoint inhibitor (in particular PD-1 and TIM-3 antagonists) based on the conclusion that the patient has a high probability to achieve a response with an immune checkpoint inhibitor when the patient has a co-existing chronic obstructive pulmonary disease [6]. Infiltration of TME by Treg cells may also lead to the dampening of the adoptive immune response against cancers. In this context, quantifying the density of Treg cells along with the density of at least one additional population of immune cells (e.g., mature dendritic cells, B cells or $\mathrm{CD}^{+} \mathrm{T}$ cells) can be particularly suitable for determining whether a patient suffering from cancer is eligible to an ICBT [7].

\section{Tools for deciphering the cellular composition of the TME}

Since, the contexture between tumor cells and their TME is clinically crucial for determining the ICI efficacy, tools allowing the deciphering of the cellular composition of the microenvironment are particularly needed. Recently, a method, named MCP-counter, has been developed and is based on transcriptomic markers that assesses the proportion of several immune and stromal cell populations in the TME from transcriptomic data [8]. The method has been validated against immunohistochemistry measures, demonstrating that it can provide an interesting alternative to classical immunoassays for analyzing the TME. Driven by the robustness provided by the methodology, several international patent applications describing and claiming the signatures of different immune cells as well as fibroblasts and vessels, and their use for predicting responses to ICI were published in 2017 [9-17]. Moreover, the MCP-counter methodology is particularly suitable for stratifying patients suffering from colorectal cancer according to the 'Consensus Molecular Subgroups (CMS)' classification that categorizes the cancer in four classes: CMS1-CMS4. For instance, CMS1 that includes microsatellite unstable patients, is the best prognostic group for disease-free survival and is characterized as showing the best responses to anti-PD1 antibodies. In this category of colorectal cancer, overexpression of several checkpoints was associated with a poorer prognosis independently from tumor stage and despite concomitant high expression levels of CTL/Th1/cytotoxicity markers [18]. These findings suggest that immune checkpoints, and in particular the druggable PD-1, PD-L1, LAG-3, TIM-3 and IDO molecules, may have a dominant impact above other immune components for predicting responses to ICBT [19].

\section{Genetic markers}

Genetic instability, gene expression changes, and the resulting heterogeneity within the tumor cell populations have all been associated with the process of invasion and metastasis. In this context, it has been shown that mutations of the $F B X W 7$ gene were associated with the absence of metastasis in patients suffering from colorectal cancer and this profile was explained by the fact that the mutations correlate with a strong immune adoptive response in the primary tumor with augmented immune checkpoint molecules expression. The presence of a FBXW7 mutation was thus patented as a biomarker for predicting the ICI efficacy [20].

\section{Conclusion}

The advent of ICI has led to a paradigm shift in the field of cancer treatment. However, the development of robust biomarkers to provide prediction of response and clinical benefits of those therapies is extremely important. Since, the therapeutic activity of ICIs is the result of a complex interplay between multiple factors in the TME, a sound analysis of said environment is thus of particular importance. Accordingly, assays allowing the deciphering of the contexture of the TME can thus represent accurate tools for predicting the responses to ICIs. This explains why an extensive activity for patenting said methods was recently developed. Since the pipeline of new ICIs is absolutely profuse, the race for patenting new biomarkers will certainly increase again in the future.

Financial \& competing interests disclosure

The author has no relevant affiliations or financial involvement with any organization or entity with a financial interest in or financial conflict with the subject matter or materials discussed in the manuscript. This includes employment, consultancies, honoraria, stock ownership or options, expert testimony, grants or patents received or pending, or royalties.

No writing assistance was utilized in the production of this manuscript. 


\section{References}

1. Hanahan D, Coussens LM. Accessories to the crime: functions of cells recruited to the tumor microenvironment. Cancer Cell 21, 309-322 (2012).

2. Pardoll DM. The blockade of immune checkpoints in cancer immunotherapy. Nat. Rev. Cancer 12, 252-264 (2012).

3. Herbst RS, Soria JC, Kowanetz M et al. Predictive correlates of response to the anti-PD-L1 antibody MPDL3280A in cancer patients. Nature 515, 563 (2014).

4. INSERM (Institut National de la Santé et de la Recherche Médicale), Université Paris Descartes, Assistance Publique Hôpitaux De Paris (APHP). WO2014023706 (2014)

5. Assistance Publique Hôpitaux de Paris (APHP), Institut National de la Sante et de la Recherche Médicale (INSERM), Université Paris Descartes (Paris 5), EP2872646B1 (2017).

6. INSERM (Institut National de la Santé et de la Recherche Médicale) Medimmune, Llc Université Paris Descartes, Université Pierre Et Marie Curie (Paris 6), Université Paris Diderot - Paris 7, WO2017202962 (2017).

7. INSERM (Institut National de la Santé et de la Recherche Médicale), Université Paris Descartes, Université Pierre Et Marie Curie (Paris 6), Université Paris Diderot - Paris 7, Assistance Publique-Hôpitaux De Paris (APHP), WO2017032867 (2017).

8. Becht E, Giraldo NA, Lacroix L et al. Estimating the population abundance of tissue-infiltrating immune and stromal cell populations using gene expression. Genome Biol 17, 218 (2016).

9. INSERM (Institut National de la Santé et de la Recherche Médicale), Université Paris Diderot - Paris 7Université Pierre Et Marie Curie (Paris 6), Université Paris Descartes, Ligue Nationale Contre Le Cancer, Assistance Publique-Hôpitaux De Paris (APHP), WO2018011166 (2018).

10. INSERM (Institut National de la Santé et de la Recherche Médicale), Université Paris Diderot - Paris 7Université Pierre Et Marie Curie (Paris 6), Université Paris Descartes, Ligue Nationale Contre Le Cancer, Assistance Publique-Hôpitaux De Paris (APHP), WO2017055319 (2017).

11. INSERM (Institut National de la Santé et de la Recherche Médicale), Université Paris Diderot - Paris 7Université Pierre Et Marie Curie (Paris 6), Université Paris Descartes, Ligue Nationale Contre Le Cancer, Assistance Publique-Hôpitaux De Paris (APHP), WO2017055320 (2017).

12. INSERM (Institut National de la Santé et de la Recherche Médicale), Université Paris Diderot - Paris 7Université Pierre Et Marie Curie (Paris 6), Université Paris Descartes, Ligue Nationale Contre Le Cancer, Assistance Publique-Hôpitaux De Paris (APHP), WO2017055321 (2017).

13. INSERM (Institut National de la Santé et de la Recherche Médicale), Université Paris Diderot - Paris 7Université Pierre Et Marie Curie (Paris 6), Université Paris Descartes, Ligue Nationale Contre Le Cancer, Assistance Publique-Hôpitaux De Paris (APHP), WO2017055322 (2017).

14. INSERM (Institut National de la Santé et de la Recherche Médicale), Université Paris Diderot - Paris 7Université Pierre Et Marie Curie (Paris 6), Université Paris Descartes, Ligue Nationale Contre Le Cancer, Assistance Publique-Hôpitaux De Paris (APHP), WO2017055324 (2017).

15. INSERM (Institut National de la Santé et de la Recherche Médicale), Université Paris Diderot - Paris 7Université Pierre Et Marie Curie (Paris 6), Université Paris Descartes, Ligue Nationale Contre Le Cancer, Assistance Publique-Hôpitaux De Paris (APHP), WO2017055325 (2017).

16. INSERM (Institut National de la Santé et de la Recherche Médicale), Université Paris Diderot - Paris 7Université Pierre Et Marie Curie (Paris 6), Université Paris Descartes, Ligue Nationale Contre Le Cancer, Assistance Publique-Hôpitaux De Paris (APHP), WO2017055326 (2017).

17. INSERM (Institut National de la Santé et de la Recherche Médicale), Université Paris Diderot - Paris 7Université Pierre Et Marie Curie (Paris 6), Université Paris Descartes, Ligue Nationale Contre Le Cancer, Assistance Publique-Hôpitaux De Paris (APHP), WO2017055327 (2017).

18. Marisa L, Svrcek M, Collura A et al. The balance between cytotoxic T-cell lymphocytes and immune checkpoint expression in the prognosis of colon tumors. J. Natl Cancer Inst. 110(1), doi:10.1093/jnci/djx136 (2018).

19. INSERM (Institut National de la Santé et de la Recherche Médicale), Université Pierre Et Marie Curie (Paris 6), WO2017114851 (2017).

20. INSERM (Institut National de la Santé et de la Recherche Médicale), Université Pierre Et Marie Curie (Paris 6), Université Paris Diderot - Paris 7, Université Paris Descartes, WO2017103086 (2017). 
